\title{
The Long and Dramatic History of Surgical Infections
}

\section{Sergio Sabbatani ${ }^{1}$, Fausto Catena ${ }^{2}$, Luca Ansaloni ${ }^{3}$, Massimo Sartelli ${ }^{4}$, Belinda De Simone ${ }^{2 *}$, Federico Coccolini ${ }^{3}$, Salomone Di Saverio ${ }^{5}$ and Antonio Biondi ${ }^{6}$}

${ }^{1}$ Department of Infectious Diseases, St Orsola-Malpighi University Hospital, Bologna, Italy

${ }^{2}$ Department of Emergency and Trauma Surgery, Parma University Hospital, Parma, Italy

${ }^{3}$ Department of General and Emergency Surgery, Papa Giovanni xxiii Hospital, Bergamo, Italy

${ }^{4}$ Department of General Surgery, Macerata Hospital, Macerata, Italy

${ }^{5}$ Department of General Surgery, Maggiore Hospital of Bologna, Bologna, Italy

${ }^{6}$ Department of General Surgery, Catania University Hospital, Catania, Italy

${ }^{*}$ Corresponding author: De Simone B, Department of Emergency and Trauma Surgery, Parma University Hospital, Parma, Italy, Tel: 393200771984; E-mail: desimoneb@hotmail.it

Received date: October 26, 2016; Accepted date: November 07, 2016; Published date: November 14, 2016

Citation: Sabbatani S, Catena F, Ansaloni L, Sartelli M, De Simone B, et al. The Long and Dramatic History of Surgical Infections. Arch Med. 2016, $8: 6$

Copyright: (c) 2016 Sabbatani S, et al. This is an open-access article distributed under the terms of the Creative Commons Attribution License, which permits unrestricted use, distribution, and reproduction in any medium, provided the original author and source are credited.

\section{Abstract}

Background: Surgical infections cause still significant morbidity and mortality all over the world, nevertheless new surgical techniques and advancements in the treatment of patients affected by primary and secondary peritonitis.

The aim of this historical review is to retrace the history of the fight against the iatrogenic infections in surgery from XVIII to XX century.

Materials and Methods: We carried a systematic historical review about all the available documents about surgical infections and the development of aseptic method in surgery.

Results and Conclusions: Discoveries by discoveries we found personality as Malgaigne, Spallanzani, Lister and many others scientists working for understand the etiopathological process responsible of sepsis. Analyzing their studies, it is evident how many factors can influence this disease and how many solutions proposed in the past are utilized even now.

Keywords: Surgery; Asepsis; History of surgery; Wound care; Surgical infection

\section{The History of Asepsis}

In an essay dedicated to Ignazio Semmelweis, the celebrated author Luis Ferdinand Cèline writes that during the Pasteur era, nine of ten surgeries ended in death due to infection [1].
The first reliable statistics on operative mortality were published in 1841 by the Frenchman Malgaigne (1806-1863). They noted that the average mortality of amputations was $60 \%$ and was primarily caused by hospital diseases [2]. Despite the general term of "hospital diseases", five clinical entities were diagnosed with significant frequencies: erysipelas, tetanus, pyemia, septicemia, and nosocomial gangrene. In the case of pyemia, it is worthwhile to remember that physicians were aware that pus exiting the putrid wounds into larger vessels suddenly caused the beginning of illness marked by chills and high fever. Furthermore, autopsies in these cases consistently demonstrated numerous purulent metastases in internal organs. Septicemia was understood differently, in the sense that its development was slower than pyemia, and was manifested in the putrefaction of blood and the resulting secretion from the wound assuming a grey-brown color. Unlike pyemia, the region of the infection remained minimally sensible and painful in septicemia. In the case of nosocomial gangrene, an adherent and oily coat first covered, and then slowly penetrated, the wound $[2,3]$.

Most surgeons, and general physicians of the time period understood suppuration, and the production of pus, as a normal, inevitable process along the road to scarring. Common practices included administering medications by inserting dirty, yarn-like material into wounds with filthy instruments. Furthermore, surgeons would use the fibers of whips, bought in stores meant for cart drivers, to tie-off procedures. Finally, the surgeons themselves wore very old gowns to perform procedures, often times encrusted with dried fluids and human material from previous operations. In fact, these gowns were a point of pride, indicating a long career in the operating room [4]. All these alone could explain the high mortality in hospitals. 
According to Grmek, serious complications caused by purulent inflammations were few in medieval times and became more common throughout the Renaissance and quite frequent in the centuries that followed [5]. Retrospective analysis has shown that this time period was marked by a significant increase in the virulence of pyogenic bacteria combined with the natural variation of germs and general deterioration of social factors. Within these social factors it is important to remember the general increase in human concentrations: overly-populated living spaces, development of large hospitals with communal waiting areas for patients carrying all different types of diseases, and the creation of large, permanent militaries. Common practices by surgeons, such ignoring the importance of clean hands, instruments, and clothing during interventions, all contributed to the risk of serious infection. The increase in wounds caused by fire-arms, in which gunpowder residue often infiltrated the opening, also played an important role in the risk of infection [5] according to many notable figures, including Braunschweig (1450-1512), Giovanni da Vigo (1450/60-1520/5), Alfonso Ferri (1515-1595), and Clowes (1540-1604). Maggi (1476-1552), Lange, Jobert (1529-1583), Fabry (Fabricius) (1564-1634), Magati (1579-1647) and Paré (1510-1590) were of the opposite opinion, believing that lesions caused by firearms were of the same nature caused by non-firearms and should be treated with conservative methods [6].

In the $18^{\text {th }}$ century an aggravation of wounds was detected, but the cause remained unclear. During this time period the adjective "putrid" was used to determine infections and putrid fevers referred to tetanus, pyemia, and septicemia. These fevers were seen in the wounded, in patients who had undergone surgical procedures, in mothers who had just given birth, and in big cities, in hospitals (hospital gangrene). Towards the end of the $18^{\text {th }}$ century, in large cities such as London, Paris, and Vienna, many hospitals had an influx of diseases that resulted in precarious sanitary conditions and an increase in operational risk $[5,6]$. This increase was also linked to the growing practice in Paris and later also in Vienna, of performing autopsies in the rooms used for operations [3] thus causing an increase in nosocomial infections. During the 1840's visiting mothers who had just given birth immediately after autopsies were performed in the same rooms resulted in a significant increase in fevers experienced by these patients [7]. During this time period it was also common to complete surgeries as fast as possible, as it was thought that this would reduce the risk of febrile illnesses, in addition to demonstrating the surgeon's ability. In reality, this practice led to dangerous hemorrhage, as surgeons would only close large vessels, leaving the smaller ones to bleed internally. Blood was collected with sponges that were washed with cold water between surgeries.

Several statistics from the middle of the $19^{\text {th }}$ century can also be useful in understanding operating risks. Syme (1799-1870) calculated a mortality rate of amputees in Edinburgh (43\%), Boston (26\%), and London (23\%). In Munich, nosocomial gangrene in clinical surgery reached $80 \%$. In the cantonal hospital of Zurich, $46 \%$ of amputees did not survive [3]. Due to the dramatically dismal hygiene conditions, it was common to close down specific hospital departments, and in certain cases, to evict entire hospitals. There existed a general understanding that some diseases were found predominantly in hospitals and that patients who elected to be operated in their own homes were more often than not spared from these specific pathologies.

Along these lines followed an experiment by the Russian surgeon Nicolai Iwanowitsch Pirogoff (1810-1881). Around 1860, he brought home patients who had recently been operated on in his hospital, and despite laying down and recovering on beds made of dirty straw, these patients rarely become infected with the aforementioned hospital illnesses.

According to the Londoner surgeon Erchsen (1818-1896) the best way to combat an influx of cases of pyemia in hospitals was to operate using a pickax, similar to the procedure used against the bovine plague, which was eradicated using surgeries involving axes [3].

The operating room at the University College of London consisted of an old, rough wood table, a small closet with few instruments including a couple of large knives and tweezers with wooden handles, and a sink to was one's hands and to attach an opening for gas. There was also a table with the necessities for anesthesia. Billroth mentions that his operating room in Vienna was used for necropsies in early mornings followed by operations on patients on the same operating table and often times, with the help of the same nurse.

These abysmal hygienic conditions in operating rooms led the surgeon James Young Simpson (1811 - 1870) to comment, "The patient lying on our operating room table has a greater risk of death than an English soldier on the battlefield in Waterloo!" [2]

The publication of Semmelweis' (1818-1865) book, "The etiology, the concept and the prophilaxis of puerperal fever" in 1861 prompted the medical world to no longer accept nosocomial infections as an inevitable consumer of humans lives. Recounting his experiences, Semmelweis wrote about the causes of puerperal sepsis and the precautions that should be introduced to reduce it. The times were ripe for a pivotal development of experimental microbiology and application in the surgical field that would lead to antisepsis and later on asepsis. "Contagium vivum" marks the birth of microbiology $[8,9]$.

Beginning in the $16^{\text {th }}$ century, several authors hypothesized that "living microscopic beings" were involved in the contagion and ethio-pathogenesis of diseases. These hypotheses were based on theoretical reasoning and not experimental demonstration, and thus did not lead to significant progress in clinical and epidemiological knowledge [8-10].

However, at least the idea of the "contagium vivum" was present before the $19^{\text {th }}$ century and had a clear connection to the experiences of epidemics in the recent time periods, despite resistance by the majority of the medical field. The miasma theory, and its relation to putrefaction and bad odor, remained the most common way to explain contagions and infections to the common person. A commonly used example 
was that of one rotten fruit that, when in close contact with other fruits, would lead to their rottenness as well $[9,10]$.

The development of the microscope in the first three decades of the $19^{\text {th }}$ century led to important discoveries in the world of microbiology. At the beginning of the century, Amici (1786-1863) technically improved the microscope by using achromatic lenses that eliminated optic aberrations that previously had resulted in observing strange globules on prepared slides. These optic distortions had led to a curiosity and imagination of the general public of this era. During the same time period, the use of taxonomy gained popularity and acted as the foundation for the concept of continuity and permanence in morphological organization $[9,10]$.

In 1837, Belli performed the first experiment, involving the muscardine illness that affects silkworms, that sustained that a disease can be caused by a living microscopic organism. Belli believed that most infectious diseases are caused by many types of plant or animal microorganisms [11].

This discovery by Belli marked a major turning point on the biological scale. It was accompanied by a crucial change in epistemological thought, which overcame the prevailing concept of "sufficient and varied causality" and replaced it by the idea of a singular, specific causation of an infection, and thus of an illness [12]. During the first couple of decades of the 1800's Enrico Acerbi and others knew that petechial typhus was caused by "organic, parasitic beings" but they did not know the origin of these "beings".

According to Fracastoro, the "first seeds" were born by spontaneous development from "corrupted humours" and were transmitted via direct contact, impure objects, or through the air. The germs of diseases were activated by the humours, caused by a link between man and the divine creation [8].

The first to question the theory of spontaneous generation was Redi (1626-1697), who publishes his book "Experiences regarding the generation of insects" in 1668. His experiments irrefutably describe that leaving meat in sealed containers lead to its decay with no formation of other life forms, where as meat left in unsealed containers led to the creation of fly larva. This elegantly discredited the concept of spontaneous generation [13]. Paradoxically, Anton van Laenwenhoech's development of microscope techniques led to a renewed interest in the theory of abiogenesis. His work allowed for the observation of many very small "beings" with a swiveling circular body and tail (protozoa), of various colors and dimensions. These "beings" were coined "infusors" by Heinrich. Wrisberg because of their ability to move quickly within any type of liquid. These "infusors" were also discussed by Georges Luis Leclerc de Buffon (1707-1788) and led to the height of agreement with the spontaneous generation theory.

The students of Needham (1713-1781) also supported abiogenesis in their 1745 publication "new microscopical discoveries", which referred to their observations of germs in budding grains, infusions, plants, and animals. The authors wrote the germs originated from, "a generational force that produces globules that characteristically do not become generated". It is likely that this influenced Buffon who theorized that there were "organic molecules" that could transform themselves into living beings due to an "internal imprint" [14].

During the middle of the 1700's this topic become so lively that even Spallanzani (1729-1799) caught wind of it. Spallanzani did not receive a traditional scientific education and in fact, had begun by studying law before turning to math and physics. Due to financial constraints, he first taught Greek before receiving a teaching position from the University of Reggio-Emilia for physics and math, in which he focused on motion, astronomy, geology, paleontology, and the properties of the four elements.

This atypical and vast background allowed Spallanzani to develop ideas free of the cultural prejudice and blind loyalty of scholastic ideas at the time. During the first months of 1761 Spallanzani procured the second volume of Buffon's "Histoire Naturelle" and Needham's "observations microscopiques", both of which had regenerated the conversation on spontaneous generation. After studying this material, Spallanzani obtained a simple microscope and a set of vials and bottles to prepare various infusions to analyze. The object was to redo and restructure the experiments of Buffon and Needham, using different substances, animal and plant, to prepare the infusions. His first results confirmed Needham's findings, in particular the idea that the globules, after a large increase in number also diminish quite rapidly, and that the number of "infusors" is much greater than the "generational force" of the substances used in the infusions. This first cycle of research thus remained close to the position of Needham [14].

After some repetitions of the experiment, Spallanzani introduces some variations to address his growing suspicion that Needham's results and Buffon's experiments could be distorted, limited by the intrinsic methodology of their experiments. Variations included perfecting the closure of the bottles containing the infusions, more gradually raising the temperature and extending the time of boiling, as well as introducing new quantitative methods to measure temperature, pressure, and time [14].

These modifications made clear that the infusions as prepared by Buffon and Needham had indeed been exposed to external contamination, as the containers had not been properly sealed and the infusions were not heated at a high enough temperature. These new observations led Spallanzani to negate Needham's claims of "an absence of specific form and movement" and claim that the "infusors" did in fact retain a well-defined form and were capable of movement.

After four years, in 1765, Spallanzani published his findings in "Essay on microscope observations concerned with the theory of generation of Needham and Buffon". This work, which focused on his second phase of research, functioned as the foundation for Redi's thesis on ovular reproduction. Spallanzani's work received significant European recognition, and was read by the Genevan naturalist Bonnet (1720-1793) 
who opposed Needham's ideas, von Haller (1708-1777) and even by Voltaire.

A scientific feud between Needham and Spallanzani developed, despite the fact that Needham had originally appreciated Spallanzani's additions to his original experiment. In 1769, Needham publishes an article criticizing Spallanzani's experiments. One year later, Spallanzani had the opportunity to respond to his criticism while acting as appointed Professor of natural history in Pavia.

The experiments continued and addressed also the nature of spermatozoa, which had previously been seen as an emission in the first round of research. In 1776, his results are released in Modena regarding experimental observations of "vermicelli spermici" (vermicelli-like sperm). During the new cycle of research, Spallanzani accentuated the rigor of his scientific method and, in response to Needham, uses cooked and uncooked meat as substrate. Spallanzani concluded that heat alone is not enough to impede the development of the "infusors" by observing that infusions in both the cooked and uncooked meat led to the production of organisms. Critically analyzing his findings, Spallanzani suspects that germs, or eggs, could be transported via air into the infusion ampules. To test this, he proceeded to sterilize the infusion ampules by subjecting them to an even longer boiling time than done in previous rounds of research where he had followed Needham's model. Furthermore, Spallanzani flame-seals the ampules to ensure that they do not become externally contaminated. This newest set-up led to observations of no "infusors" within the ampules. The counter-experiment, in which air was introduced into the ampules, led to the opposite observation. Microscope analysis that followed confirmed the "animaluzzi" (microorganisms). Spallanzani discovered that the limitations of Needham's set-up had been the use of cork stoppers for the ampules that allowed air to penetrate inside. Given his observations, Spallanzani concluded that there was absolutely no basis for the idea of spontaneous generation of "infusors". However, Needham remained steadfast in his original opinion. He believed that Spallanzani's methods had been too aggressive, not allowing for the "natural vegetative force" to run its course. He especially criticized Spallanzani's excessive boiling to sterilize the infusion bottles. Spallanzani responded that the variations in the air had not modified the experimental parameters. This seemed to put an end to the back-and-forth. Although it was not understood at the time, Needham had brought up a valid point, as Spallanzani's extreme heating had, in fact, changed the composition of the air by removing the oxygen.

Spallanzani's methods spurred the French industrial preserver Appert (1749-1841) to propose, in 1810, a method to conserve general foodstuffs. Appert retained that heat killed the putrefying agents and introduced the technique of conserving food in hermetically closed and heated containers [15]. Later on, the work of Louise Joseph Gay-Lussac on oxygen's role in fermentation demonstrated that it was in fact the lack of oxygen, and not heat in itself, that killed the agents of putrefaction.
During the 1830's and 1840's, problems involving the conservation of food and fermentation, and in particular their economic importance due to the growing food conservation industry, led to a spark in research in this field. From this, two opposing opinions were created: the first that believed fermentation could be entirely explained by chemistry, and the second that believed that although there was a chemical nature present, the main responsibility lay with the living organisms that could be microscopically observed in every material undergoing fermentation or putrefaction $[12,16]$.

In 1837, Schwann (1810-1882) and Cagniard-Latour (17771859 ) independently discovered that the spherules of yeast previously observed by Leeuwenhoek in beer and wine during fermentation, were living organisms. This discovery proved to be important for Pasteur's future research. During the same time period, Henle (1809-1885), convinced of the importance of germs in human pathology, experimented in search of living contagions in cadavers of typhus patients and in scarlet-fevercaused skin flakes. Although he was unable to make any conclusive findings, he did write "Pathological Investigations" and detailed the postulates necessary to consider a germ the cause of an illness by writing, "[the germ] must be present in all infected individuals, it must be isolatable from the infected organism, and it must be able to infect a healthy individual" [9]. The times were ripe for the Pasteurian revolution.

Between 1857 and 1860 Pasteur released his first studies regarding lactic fermentation. Pasteur presents his opinion clearly: fermentation is the result of activity by very specific microorganisms. Each fermentation is a result of one specific microorganism that develops in specific chemo-physical and feeding environments. Particular conditions may arise which advance or restrict the microorganism's development, and a "biological fight" may be created between different microorganisms in search of the same food. Furthermore, one can manipulate the conditions to culture specific microorganisms to then be able to identify and purify them.

These initial experiments already hinted at the fact that the microorganisms responsible for fermentation are carried via air [12]. In the years that followed, Pasteur focused on alcoholic fermentation, the transformation of sugar, and the importance of yeast. He successfully cultures lactic and alcoholic fermentation, and in 1861, specifically cultures the fermentation of butterfat. In this fermentation, Pasteur microscopically observes mobile rods, which at the time were classified as animals. Furthermore, by removing all the air from the butterfat fermentation he observes a complete killing of all organisms. When he tried to do the same with carbon dioxide, no effect was noted. These experiments led to the following simple yet elegant conclusion: butterfat fermentation is caused by an "infusor" that lives in an environment lacking oxygen [17].

Pasteur went on to address spontaneous generation, the dividing question of the middle of the 19th century. Thanks to a prize received from the Academy of Sciences in Paris in 1860, Pasteur interest was piqued and he began researching this controversial topic. 
The leading figure of the time against Pasteur was Felix Pouchet (1800-1872), a naturalist from Rouen, France. Pouchet was strictly an experimental scientist, maintaining a completely empirical approach in the laboratory and ignoring any theoretical or rational basis. Pasteur on the other hand worked on the basis of strong ideological and theoretical foundations, including the ideas of anti-materialism and the necessity of the continuity of life. In his "germ theory" he elaborates on spontaneous generation by explaining his idea regarding "organic machines" that are made of a distinctive structure that cannot be generated spontaneously from a material that does not contain its structure [12]. A crucial year in the development of microbiology, in 1860 Pasteur presents to the Academy of Sciences his first findings regarding spontaneous generation.

Pasteur began his experiments by passing air through vials with filters made of cotton balls. He noticed that the cotton balls would then always contain corpuscles, whose structure was very similar to that of living organisms. He now wanted to demonstrate that these corpuscles were in fact "fertile germs", the microorganisms present in infusions. He did this by placing cotton balls filled with atmospheric dust in originally sterile containers. After one or two days, the liquid within the containers became colonized by microorganisms. In the second round of experiments, to depict the importance of the atmospheric air, Pasteur used special, long-necked containers in which he placed sterile liquid. Despite being exposed to the air by a small opening at the top of the container, the liquid remained sterile and Pasteur was able to deduce that it was not the air, but rather the corpuscles present in the atmospheric dust that contaminated the liquid.

Immediately following this, Pasteur began another round of experiments to address a major doubt posed by others regarding his findings: if such a small quantity of air was able to produce microorganisms in every type of infusion because of the presence of germs in the air, then the air had to be populated by a variety of germs and be so dense that it would resemble a fog. To answer this, Pasteur proceeded to expose the infusions to air in different locations with varying altitudes and humidity. He found maximum generation in the infusions exposed to the air in Paris, and the minimum, only one bottle out of twenty, in the frozen air of the Jura mountains in France and Switzerland. Pasteur concluded that the generation of microorganism was not due to the air in itself, but due to the germs present in the air, whose concentration depended on environmental conditions [18].

The following year, Pasteur directly addressed the work of his opposition, and suggested an experimental error in the work of Pouchet in that the mercury he had used to keep the containers sterile could have actually contaminated the samples. To prove this indisputably, Pasteur repeated Pouchet's set-up but only used mercury that had previously undergone calcination. The result was no development of microorganisms whatsoever [19].

The scientific rivalry continued and the experiments were repeated until 1864 when the Academy of Sciences appointed a panel of experts to once in for all declare a winner. Pouchet declined to present and thus Pasteur had an easy time convincing the panel of the validity of his experiments.

Although Pasteur was declared the winner, there remained some problems with the basis of his experiments. It would have been difficult to disassemble the experiments of Pouchet, but had Pasteur done so, he would have noticed that a simple introduction of pure oxygen into the Pasteur's ampules would have been enough to cause the creation of microbic cultures. Later on it was established that Pasteur used a different experimental model than Pouchet, and thus had gotten different results. Pasteur's model used beer yeast, while Pouchet's used hay yeast, in which spores can survive and develop in high temperatures and in the presence of oxygen. This led to the understanding that if Pouchet's ampules had been used in Pasteur's experiments, development of microorganisms would have resulted and sustained the argument for spontaneous generation.

\section{Crisis in Surgery and the Innovations of Joseph Lister}

As seen in the first part of this paragraph, surgery remained in a lamentable state during the middle of the $19^{\text {th }}$ century, in large part due to surgical infections. There had been attempts to control wound infections, but there remained much confusion amongst surgeons as there was no etiological evidence regarding hospital illnesses (nosocomial infections).

During the middle of the $19^{\text {th }}$ majority, sixty percent of surgical cases were comprised evenly by three interventions: amputations, tracheotomies due to diphtheria, and tumors most commonly found in superficial positions. In addition, facial plastic surgery was relatively common, pleural empyemas were seen a fair amount, and hernia removals, abdominal, and thoracic surgeries were very rarely seen [2].

One of the first exceptions to this pattern was the English surgeon Thomas Spencer Wells (1818-1897) who in 1858 performed the first ovary removal surgery. Continuing this line of work in a very accurate and scrupulously hygienic manner, Wells wrote to the Royal Society in 1881 that he had done 1000 ovarian surgeries.

Despite this example, most surgeons were still in the dark on how to limit surgical infections, and tried various approaches that unfortunately could only be based on empirical evidence and not on solid theoretical foundation.

In France the "occlusive method" was adopted to cover the wound from harmful air. Various covers, including thin layers of gold paper, natural rubber, and collodion were used to protect the wounds but all were unable to reach their objective of keeping air out as the secretions from the wound would unseal the material from the skin [2]. The surgeon JulesRené Guérin (1801-1886) built a complicated device that used rubber caps attached to a pump, which could aspire air and secretions away from amputated body parts [2].

About fifty years prior to this, at the beginning of the $19^{\text {th }}$ century, von Kern (1760-1829) saw the unsuccessful results of 
his colleagues in Vienna that tightly covered wounds with strips of cloth, tried the "open treatment" allowing wounds to be exposed to the surrounding air. This method received little attention.

Yet another method, the "incubation method" was tried by the French military surgeon Larrey (1766-1842) who, during the campaign in Egypt, noticed that the wounded faired much better in the warmer, more humid climate than during the prior Napoleonic military campaigns. Thus, Jules Guyot (1808-1872) built a thermostat that could maintain rooms for wounded soldiers at specific temperatures. For the same reason, Swiss surgeon Mayor (1775-1847) recommended hot baths for wounds. However, immediately after in 1850 in Kiel, von Esmarch (1823-1908) practiced bathing wounds in ice water.

Bouisson (1813-1884) believed that allowing wounds to dry under a steady stream of air could be the solution to the question regarding the best way to avoid infections during the wound healing process. This approach resembled that of Hunter (1728-1793), whose objective was to protect the wound by substituting the scab with purulent secretions. Hunter thought that by doing so, the wound would be protected and could heal more quickly. He did not believe that the exclusion of air played a fundamental role in wound healing but did believe that suppuration could be avoided by artificially creating a scab. In Berlin, von Langenbeck (1810-1887) agreed with Bouisson that they exclusion of air played an important role in wound care [2]. Von Langenbech adopted a warm water immersion therapy.

It was well known that closed fractures had a higher success rate in the operating room compared to open fractures in the likelihood of developing an infection. Thus a group of surgeons developed a "subcutaneous operating" method to avoid the creation of a large, open wound. John Hunter put this method into practice by operating on dogs subcutaneously, entering the skin by a small incision. After Hunter, Jacques Mathieu Delpech (1777-1832) applied this method to human surgery, which was then later expanded by Dieffenbach (1792-1847) and Stromeyer (1804-1876) [2].

Around 1860, the surgery department at the University of Glasgow, directed by Thomas Anderson, (1819-1874) was still experiencing the difficulties of surgery and very high mortality rates due to surgical infections. Along the corridors one could smell the repugnant odors emitted from wound infections. Anderson had read Pasteur's work on putrefaction and fermentation and recommended it to Lister. Lister became convinced that suppuration of wounds and putrefaction of organic substances worked in the same way, in that both were caused by germs. More specifically, Lister believed that wounds became infected by germs suspended in the air.

During this time period Lister also began a remarkable public health initiative: applying carbolic acid to wounds in the first attempts at antiseptic healing. Inspired by the use of minimally concentrated carbolic acid in the meadows surrounding the city of Carlisle to eliminate the odor of putrefaction, Lister applied cotton dipped in highly concentrated carbolic acid to wounds in hopes of forming an antiseptic crust [3]. He first applied this in 1865 in a case of an open fracture. Unfortunately, after four days the scab fell off. Lister then thought of using an entire cloth soaked in carbolic acid to generously cover the area around the wound. Even with this approach the results were poor, as the carbolic acid evaporated far too quickly to obtain a significant effect. He tried to correct this by covering the medication with wax paper and aluminum foil [3].

These experimental efforts resulted in a decrease in the cases of suppuration, in comparison to those treated with cloth strips as had been previously done. However, even the careful disinfection of the entire area around the wound with gauze soaked in carbolic acid did not completely eliminate suppuration. Lister concluded that the diluted phenol could cause what he called an "antiseptic suppuration". Thus, he decided to drain secretions and simultaneously place a protective material around the wound to reduce the irritation caused by undiluted carbolic acid. More specifically, he reduced the percentage of phenol in the soaked gauzes to five percent, added a layer of waxed taffeta as protective material, and finally placed the actual carbolic acid medication on top of this layer [3].

After this preliminary adjustment, Lister continued to improve his antiseptic medication technique. In 1868, Lister's new version focused on isolating the bloody surface of the wound from the carbolic acid by superficially applying a completely aseptic medication. Only the drained secretions came into contact with the carbolic acid medication [3]. In the year prior, Lister's first publication titled: On a New Method of Treating Compound Fracture, Abscess, etc., with observations on the condition of suppuration was published in the Lancet.

Besides working on antiseptic medication, during this time period Lister also verified several of Pasteur's experiments. He again showed that fermentation was not caused by air alone, but by germs in air by experimenting with the fermentation of urine in specially-shaped bottles. The bottleneck had been stretched and twisted in a way that when the urine contained in the bottle was boiled, the urine "trapped" in the twisted section did not ferment because it could not be colonized by the germs in the air [3-20]. Lister's innovations rested on two completely new theoretical principles: the agents of putrefaction were ubiquitous germs and infection could not be seen as a normal stage in the wound healing process [21]. Although Lister did not have experimental proof, his conclusions were based on clinical results by statistically comparing the lethality of fractures prior to the introduction of medication with carbolic acid to those in which it had been used. Results showed that cases in which medication with carbolic acid was applied had lower lethality rates. However, it has been more recently suggested that Lister had specially "selected" his cases [22] to obtain favorable results.

An important point to be remembered is the heightened administrative costs that an "antiseptic system" placed on hospitals. Furthermore, the theory of the role of germs in putrefaction led to a shift in responsibility of the results of a treatment from the vulnerability of the patient and his or her 
environment to the surgeon, who with his hands and instruments, could introduce germs into the patient's body. Finally, one has to recall the social crisis that followed the Industrial Revolution in the United Kingdom in the middle of the $19^{\text {th }}$ century, and its likely skewing of the results that showed high post-amputation mortality rates in glasgow.

Nowadays, considering the socio-economic level during the time in which Lister operated, one can object to the observation above of heightened mortality rates. According to Trohler, the positive results of Lister's experiments could also be attributed to the improved economic conditions in Glasgow that included an increase in foodstuffs, availability of running water, and new water waste management.

Lister was not satisfied with the results reached in the 1870 's and thus in the next twenty years continued to evolve his technique [23]. This led to the disinfection of not only all wounds, but also of surgical instruments, surgeon's hands, and even for some time, the air in the operating room.

Beginning in the 1870s, sterilized gowns, masks, and gloves were gradually introduced, although Lister himself believed these precautions to be unnecessary and did not utilize them in his practice. The introduction of the concept of antisepsis to the surgery world had been difficult and full of resistance. Some objections had even been malicious, as those directed towards the ideas of Semmelweis, while others proved to be worthwhile and even positive.

Some believed Lister's ideas to be somewhat unoriginal, as some had already been proposed but eventually abandoned as the theory of "open treatment of wounds" become more popular. In reality, this criticism was not pertinent as Lister was interested in excluding germs, not air, from wounds.

Others remembered the "antiseptic" practices of surgeons from several decades before. Auguste Nélaton (1807-1873) used alcohol-based medications to counteract expressions of putrefaction emitted from wounds. In the United Kingdom, medications based on glycerin were used for the same purpose. In France in 1773, Louis-Bernard Guyton de Morveau (1737-1816) discovered the disinfectant properties of chlorine and advocated for chlorine fumigations of hospitals to kill the miasmas in the air. In 1825, Labarraque (1777-1850) created a solution of sodium hypochlorite that he sustained was very effective. In 1831, gynecologist Karl Christofer Hueter (1803-1857) suggested the use of chlorine-based water. A bit later, in 1859, the French surgeon Velpeau (1795-1867) introduced the antiseptic use of iodine.

The most pointed criticism came from the prestigious scientist and English surgeon James Young Simpson (1811-1870). He had gone against the ideas of Semmelweis and had created his own method of wound treatment. Simpson vocally disagreed with Lister, stating that Lister ignored literature relevant to wound treatment and antiseptic care. It was true in fact that Lister did not know of all the literature on this topic and that a convincing opinion remained in the air in Europe at the time.
In 1815, Chaumette observed that tar from fossil fuel had antiseptic properties. Runge discovered carbolic acid in 1834 which, combined with naphthalene, gasoline, paraffin oil, and aniline formed a plaster-like substance called coal tar that for a period of time was used on wounds for its antiseptic property. Coal tar was then abandoned as it left substantial residues on the wound that proved difficult to remove.

As mentioned by the Italian, Bottini (1835-1903), who had begun using carbolic acid in 1863 actually before Lister, this antiseptic would continue to be utilized long after its discovery [24]. The pharmacist Francois-Jules Lemaire observed in 1860 the ability of carbolic acid to destroy microorganisms without endangering fermentative processes. Empirical evidence also determined that in scars left behind from vaccinations that had been treating with carbolic acid, no pustule would form. Lemaire deduced that one could avoid suppuration of wounds by treating them with tar emulsions of fossil fuel or with carbolic acid. These observations led to his experimentation on dogs and later also on humans. Bottini tells us that, "in 1861, a worker from Manchester, named Calvert, mentioned carbolic acid to the Academy of Sciences in Paris in hopes that the committee in charge of disinfectant analysis would look into recommending a better disinfectant. The proposal was not immediately taken into consideration and received the opposition of Velpeau, who "without even bothering to experiment the action of carbolic acid, declared its functionality analogous to coaltar, sharing its same disadvantages" [24].

In 1862 carbolic acid was presented at the International Exposition in London and a friend of Bottini, Professor Pavesi, begged Bottini to come to Italy to "experiment (with carbolic acid) in surgery and in particular in cases of gangrenous wounds". At first, Bottini hesitated, as there was suspicion that carbolic acid could present the same difficulties as coaltar as there was a "deficiency in the proportions and use of this novel topical medication". However he adds that he "became deaf to the concerns that Pavesi would bring up" [24]. In 1863, Lemaire published a monography about carbolic acid [25]. Bottini read this to, "form a clear understanding of its action on humans, and prepare himself for the first experiments with it on humans" [24]. Bottini then writes that, "initially there were poor case studies to attempt experimentation", but then soon after was called to an important surgery department, and having been reassured by the hospital pharmacist of the purity of the medication, he began treatments. These treatments were monitored by over 600 nurses, "who diligently monitored the changes that occurred in the wounds treated with carbolic acid, comparing them to wounds treating with other substances and trying to understand what progress in wound healing could be attributed to the carbolic acid" [24]. Bottini used carbolic acid for two reasons: to disinfect "gangrenous wounds" and to moderate the process of suppuration.

Lemaire's publication in 1863 does not change the importance of Lister's work, especially with the knowledge that the English scientist had understood the strategic dimension of the problem. Almost immediately, Lister had begun to research finding a material for sutures that could be 
reabsorbed by the body. At the time it was commonplace to take out of the wound the thread used for stitching, as this provided an optimal drainage system for the copious suppuration which in the pre-antiseptic era accompanied most wounds. Lister understood the importance of having a secure suture material and studied hard to bring about the catgut [3].

Although difficult to develop, the "spray" that allowed for the pulverization of carbolic acid became a fundamental player in antiseptic treatment beginning in 1871. Using a hand pump, the vaporized carbolic acid could be applied to the wound region and to the hands and instruments of the surgeon. This application was to deter infection brought about by the germs in the air around the wound. Negative aspects of this application included the ensuing haziness of the viewing field and the mucous irritation caused by the spray [3]. Furthermore, the hands of the surgeons were also damaged by the spray, and Lister's apprentices soon abandoned the practice. After it was demonstrated that the germs in the air were innocuous in 1887, Lister himself set the spray aside.

Lister only partially accomplished his goal of inhibiting the development of germs in healing wounds. There is the limitation to consider that initially Lister applied his method only to open fractures and to patients that had been recovering in the hospital for a significant amount of time after their initial trauma. These were particularly difficult patients. Retrospectively, it is difficult to understand why Lister did not apply his method to "ideal" patients, such as amputees. The understanding of the time did not encompass the rapidity with which infectious germs could replicate and reach deep tissues in open fractures.

The second group of patients that received antiseptic treatments was carriers of abscesses. Those with tuberculosis abscesses were excluded from treatment. This detail also helps understand the limits to the development of asepsis. Already in 1878, the surgeon Billroth noted that in his opinion, Lister's method was ideal for recent wounds that had not been already infected and that chronic suppuration was not the ideal pathology for treatment with carbolic acid.

\section{The Diffusion of the Antiseptic Method}

After the publication of Lister's first work in 1867, Thiersch (1822-1895) applied antiseptic treatment in his clinic in Leipsig and obtained good results. In the coming years he made several modifications to the treatment, including using salicylic acid instead of carbolic acid.

An interesting methodological change to the original treatment was instituted by Socin (1837-1899) with his work on 20 patients. He treated another set of 20 patients with other wound treatments and compared the results with the first 20 treated with Lister's method. Those treated with Lister's method obtained excellent results whereas only 7 of the 20 cases in the alternative treatment set became free of infection. In 1872, Socin underscored that the ideal wounds to receive antiseptic treatment were those that were recent and caused by operation, not trauma. The worst results came from patients who had gone through a long waiting period between wound formation and beginning of treatment [22].

In 1881, Burckhardt (1853-1905) published the results of the last ten years in the clinic in Basel, Switzerland. Mortality rates had declined from $43.7 \%$ to $11.5 \%$ in cases of amputation, $52.7 \%$ to $10 \%$ in open fractures, and $77.7 \%$ to $10.2 \%$ in hernia removal surgeries. In amputation operations, the average time of recovery decreased from 103.5 to 48.7 days and the percentage of infection of the operational wound from $35.9 \%$ to $7 \%$ [22].

German surgeons were the most ready and the most diligent in following Lister's method, even though Berliner Bernhard von Langenbeck only began treating open fractures in 1875, after a meeting with Lister. Lister's most fervent followers included Richard von Volkmann (1830-1889) who in 1872 introduced the method in the Halle clinic and Nussbaum who adopted antisepsis in his clinic in Munich, where surgical infections had killed many patients.

In Berlin in 1870 Bardeleben (1819-1895) began implementing the method with good results and in 1872 even sent his assistant to Edinburgh to perfect the method from Scottish doctors. Thanks in part to the publication at the $3 \mathrm{rd}$ Congress of Surgery in Germany in 1874 of the two cases of Halle and Berlin, there was a vast diffusion of antiseptic treatment all throughout Germany. In the United Kingdom however the opposition to antiseptic treatment remained persistent, as was seen in the writing of Beck (1843-1893) that said, "it is an ungrateful task trying to advocate for antisepsis to a Londoner audience". Even in 1879, the important surgeon Savory (1826-1895) declared himself against the antiseptic method at a reunion of British doctors in Cork. In France, the method found support only after 1876 thanks to literature written in its support by Championnière (1843-1913).

Vienna was probably the European capital most closed minded to antiseptic treatment. In 1877, Neudorfer (1825-1898) utilized the same arguments made by Simpson to attack Lister's method. Theodor Billroth despite having admitted the theoretical weaknesses of Lister's method, began a series of experiments that led to interlocutory conclusions. Adoption of the method was initially integral, but Billroth began observing with noticeable frequency the appearance of carbolic intoxication, a phenomenon that had been rarely noticed in other clinics, and completely unnoticed by Socin in Basel. Billroth felt that Lister's continuous adjustments and improvement of his method had led to some confusion in its understanding by other doctors. Furthermore, the brown splotches left on the hands of surgeons from the carbolic acid left the surgeons feeling embarrassed and dirty [26]. Around 1875, Billroth and Lister met in Vienna and Billroth snidely asked Lister, "if (Billroth) would come to him in Edinburgh and told [Lister] that he had developed a more secure method of treating wounds, would he refuse to hear it?" Lister responded, "no". [26]. Although Billroth was convinced that the "open treatment" method was preferable over that of Lister, he courteously decided to drop the argument. 
Lister's participation in the international medical conference in Philadelphia in 1876 allowed for the diffusion of his method in the United States. However, antisepsis had already been experimented with in the US, beginning in 1868 with mixed results. Beginning in 1877, Lister becomes director of the surgery department at the King's College Hospital in London.

The Franco-Prussian war, as all wars, led to an increase in wounds and consequently a very busy time for military surgeons. During this time, the use of phenol increased, and unfortunately Lister's technique was not well known amongst all military surgeons. Furthermore, in the precarious conditions of military campaigns, it was difficult to apply with the same attention and precision that Lister had used. This then led to poor results that reflected negatively on the method that required scrupulous attention to obtain good results. Even in the hands of experts, the precarious conditions led to its failure. Poor results were found even in hospitals behind front lines, as the injured had to travel long distances in poor conditions, allowing for germs to develop difficult to control suppurations. Literature by military surgeons did not paint the treatment in a positive light, thanks to the poor results they saw in the field. Even Langenbeck and Socin, who knew the method well and had even experienced significant success, remained perplexed. Especially Socin had understood that to obtain good results antiseptic treatment had to be applied to very new wounds, in particular those caused by operation and not trauma. These same concerns and difficulties were seen by their French counterparts. The English surgeon William MacCormac (1836-1901) also expressed his doubts while working in hospitals in Metz, France and saw cloths bathed in carbolic acid being used. When he himself used this method he experienced modest results and skeptically wrote that maybe only internal use or inhalation of carbolic acid could be useful against pyemia. When Stromeyer translated the work of MacCormac, he included an appendix that was quite negative regarding Lister's method [26].

In the end, the Franco-Prussian war did not lead to the development of antiseptic treatment. Times in the medical and surgical world were not mature enough, and an understanding of microbiology was still lacking. In addition to the organizational conditions, emerging difficulties due to new weapons of extraordinary power and efficiency created extremely large obstacles for military doctors and hospitals.

\section{References}

1. Cèline LF (2007) Dr. Semmelweis adelphi edition.

2. Von Bruun-Fahrni R (1950) The wounds management and the surgical technique in the mid-nineteenth century. Rivista Ciba 24: $770-778$

3. Von Bruun-Fahrni R (1950) Joseph Lister and the aseptic treatment of wounds. Rivista Ciba 24: 779-784.
4. de Fr, Magati C (1947) On rare medicatione wounds. Rivista Ciba 1: 39 .

5. Grmek MD (1996) The hand, instrument of knowledge and therapy. In: history of western medical thought.

6. Dall'Osso E (1956) The management of gunshot wounds and its evolution in the XV-XVII centuries.

7. Semmelweis IPh (1861) The etiology, the concept and the prophylaxis of childbirth fever, pest-wien-leipzig.

8. Grmek MD (1996) The concept of disease. In: History of western medical thought.

9. Von Bruun-Fahrni R (1950) Bacteriology of wound infections and birth of the modern aseptic method. Rivista Ciba 24: 791-798.

10. Mollareth HH (1996) The great scourges. In: History of western medical Thought.

11. Pazzini A (1957) The historical background of the Bassi's discovery. Pages of medical history 1: 3-15.

12. Fantini B (1996) Medical microbiology. In: Hystory of the western medical thought.

13. Bernardi W (1980) Philosophy and life sciences. Animal generation from cartesio to spallanzani.

14. Mancini R, Nigro M, Ippolito G (2007) Lazzaro spallanzani and the refutation of the theory of spontaneous generation. Inf med 3: 199-206.

15. Appert N (1991) The art of preserving, for several years, all the animal and vegetable substances.

16. Fruton J (1972) Molecules and life: Historical essays on the interplay of chemistry and biology, Wiley interscience, New York, USA.

17. Pasteur $L$ (1861) Infusoric animals living without free oxygen gas and determining fermentations.

18. Pouchet FA (1859) Heterogeneity or about spontaneous generation.

19. Pasteur $L$ (1861) Memoir on the organized corpuscles that exist in the atmosphere. Annals of Natural Sciences 16: 5-98.

20. Chast F (1996) History of Western medical Thought. From the romantic age to the modern medicine.

21. Trohler V (1996) The triumph of surgery. In: History of western medical thought.

22. Hamilton D (1982) The nineteenth century surgical revolution: antisepsis or better nutrition? Bull Hist Med 56: 30-40.

23. Lawrence C, Dixey R (1992) Practising on principle: Joseph Lister and the germ theories of disease. In: Medical Theory, surgical Practice.

24. Bottini E (1866) Carbolic acid in surgical and taxidermic practice surgery. Univ Annals of Med 12: 585-636.

25. Lemaire FJ (1863) About phenic acid, its action on vegetables, animals, etc.

26. Von Bruun-Fahrni R (1950) The listerian method in surgery of thet period. Rivista Ciba 24: 785-789. 